\title{
Geometric interpretation of density displacements and charge sensitivities
}

\author{
ROMAN F NALEWAJSKI \\ Faculty of Chemistry, Jagiellonian University, R. Ingardena 3, 30-060 Cracow, Poland \\ e-mail: nalewajs@ chemia.uj.edu.pl
}

\begin{abstract}
The "geometric" interpretation of the electronic density displacements in the Hilbert space is given and the associated projection-operator partitioning of the hardness and softness operators (kernels) is developed. The eigenvectors $\mid \not{A}=\{|\alpha\rangle\}$ of the hardness operator define the complete (identity) projector $\hat{\mathrm{P}}=\sum_{\alpha}|\alpha\rangle\langle\alpha|=1$ for general density displacements, including the charge-transfer (CT) component, while the eigenvectors $|i\rangle=\{|i\rangle\}$ of the linear response operator determine the polarizational $P$-projector, $\hat{\mathrm{P}}_{P}=\sum_{i}|i\rangle\langle i|$. Their difference thus defines the complementary CT-projector: $\hat{\mathrm{P}}_{C T}=1-\hat{\mathrm{P}}_{P}$. The complete vector space for density displacements can be also spanned by supplementing the $P$-modes with the homogeneous CT-mode. These subspaces separate the integral (normalization) and local aspects of density shifts in molecular systems.
\end{abstract}

Keywords. Density displacements; charge sensitivites; vector space.

\section{Introduction}

The emergence of the modern density functional theory $(\mathrm{DFT})^{1-3}$ has provided a convenient framework and generated a new impetus for formulating new, thermodynamic-like approaches to classical problems in the theory of chemical reactivity. ${ }^{2,4-10}$ For example, the origin of the chemical bonding, the identity of bonded atoms, factors determining the nature and relative importance of alternative reaction sites and pathways in large reactive and catalytic systems, the stability of molecular charge distributions, similarity of molecules, the electron localization, etc., have all been approached afresh from the density perspective.

This conceptual DFT development has had a unifying influence on the reactivity theory. It combines the so-called electron following (EF) and electron preceding (EP) descriptions of both the closed and open molecular or reactive systems. In the former the nuclear displacements or the extra presence of other external potential sources in the molecular environment, e.g., due to the reaction partner, induce the redistributions of electrons, in the spirit of the BornOppenheimer (BO) approximation. This approach thus defines the electron response (chemical softness) representation, in which the external potential descriptors constitute the independent local stateparameters. The EF perspective attributes the primary role to the probing shifts in nuclear positions, i.e., changes in molecular geometry. They represent the independent perturbations, which induce the subsequent responses in the electronic structure.

However, for the complete theoretical framework, capable of tackling all issues in the theory of chemical reactivity, one also requires the complementary nuclear response (chemical hardness) representation, in which the roles of the nuclear and electronic local state-variables are reversed. In such a description one is interested in Taylor expansions in terms of the density displacements (perturbations), which are regarded as preceding the subsequent movement of the nuclei. Therefore, the electron density, the principal DFT state-variable, constitutes the independent stateparameter, to which the nuclear (geometrical) factors respond. This approach is in the spirit of the familiar Hellmann-Feynman theorem, which states that the quantum mechanical electron density uniquely determines the forces acting on the system nuclei. Such an attitude is also close to the chemical thinking about how to manipulate molecules, in order to induce desirable changes in the reactive system. Indeed, chemists often successfully modify the pattern of chemical bonds in molecules by judiciously designing a crucial electronic perturbation of the system, which ultimately induces the desirable conjugated movement of the nuclei.

It is customary to view the molecular density displacements as the resultant effect of the intermediate polarization $(P)$ component in the mutually closed subsystems, for their fixed average numbers of elec- 
trons, and the subsequent charge-transfer (CT) component in the mutually open subsystems. ${ }^{4-6}$ Therefore, for a better understanding of these intermediate stages in chemical reactivity it is vital to extract the corresponding $P$ and $\mathrm{CT}$ contributions to global and local reactivity indices, e.g., the Fukui Function or the hardness and softness kernels, ${ }^{2-10}$ which determine the transformations between the perturbations and responses in the EF and EP perspectives, respectively. In this paper we adopt the geometric strategy ${ }^{11}$ to approach this general problem, which uses the projection operators acting in the Hilbert space spanned by the independent density displacement modes in the molecule. ${ }^{4,5,11,12}$

\section{Hilbert space of independent density displacement modes}

The shift $\Delta \rho(\boldsymbol{r}) \equiv\langle\boldsymbol{r} \mid \Delta \rho\rangle$ in the equilibrium distribution of electrons in a molecule has a transparent "geometrical" interpretation in the Hilbert space spanned by the independent density displacement modes (DDM), ${ }^{11}$ in which it is represented by the vector $|\Delta \rho\rangle$. This vector space, complete for expanding a general $|\Delta \rho\rangle$, consists of the polarizational $(P)$ and charge-transfer (CT) subspaces, defined by the complementary projection operators $\hat{\mathrm{P}}_{P}$ and $\hat{\mathrm{P}}_{C T}$ respectively,

$$
\hat{\mathrm{P}}=\hat{\mathrm{P}}_{C T}+\hat{\mathrm{P}}_{P}=1 \text {. }
$$

The $P$-subspace is spanned by the orthonormal $P$ modes $|i\rangle=\{|i\rangle\},\langle j \mid i\rangle=\delta_{i, j}$, or their position representations $\boldsymbol{b}(\boldsymbol{r})=\langle\boldsymbol{r} \mid \boldsymbol{i}\rangle=\left\{b_{i}(\boldsymbol{r})=\langle\boldsymbol{r} \mid i\rangle\right\}$, which conserve the overall number of electrons $N$ in the system as a whole:

$$
\int b_{i}(\boldsymbol{r}) \mathrm{d} \boldsymbol{r}=0, \quad i=1,2, \ldots
$$

These polarization functions are the eigenvectors of the density linear response (LR) kernel, ${ }^{4,5,11,12}$

$$
\beta\left(\boldsymbol{r}, \boldsymbol{r}^{\prime}\right)=\left(\frac{\partial \rho\left(\boldsymbol{r}^{\prime}\right)}{\partial v(\boldsymbol{r})}\right)_{N} \equiv\left\langle\boldsymbol{r}|\hat{\beta}| \boldsymbol{r}^{\prime}\right\rangle,
$$

where $v$ denotes the external potential due to the nuclei, $N=\int \rho(\boldsymbol{r}) \mathrm{d} \boldsymbol{r}$ is the system overall number of electrons, and $\hat{\beta}$ stands for the underlying operator in the Hilbert space: ${ }^{11}$

$$
\int \beta\left(\boldsymbol{r}, \boldsymbol{r}^{\prime}\right) b_{i}\left(\boldsymbol{r}^{\prime}\right) \mathrm{d} \boldsymbol{r}^{\prime}=\beta_{i} b_{i}(\boldsymbol{r})
$$

or

$$
\begin{aligned}
\hat{\beta}|i\rangle & =\beta_{i}|i\rangle \text { and }\langle i| \hat{\beta}=\beta_{i}\langle i|, \\
\langle i \mid j\rangle & =\int b_{i}^{*}(\boldsymbol{r}) b_{j}(\boldsymbol{r}) \mathrm{d} \boldsymbol{r}=\delta_{i, j}, i, j=1,2, \ldots .
\end{aligned}
$$

In terms of the eigensolutions $|i\rangle$ and $=\left\{\beta_{i}\right\}$ the density LR operator is given by its spectral representation

$$
\begin{aligned}
\hat{\beta} & =\sum_{i}|i\rangle \beta_{i}\langle i| \quad \text { or } \\
\beta\left(\boldsymbol{r}, \boldsymbol{r}^{\prime}\right) & =\left\langle\boldsymbol{r}|\hat{\beta}| \boldsymbol{r}^{\prime}\right\rangle=\sum_{i} b_{i}(\boldsymbol{r}) \beta_{i} b_{i}^{*}\left(\boldsymbol{r}^{\prime}\right) .
\end{aligned}
$$

The associated projection operator

$$
\begin{aligned}
\hat{\mathrm{P}}_{P} & =\sum_{i}|i\rangle\langle i| \quad \text { or } \\
\left\langle\boldsymbol{r}\left|\hat{\mathrm{P}}_{P}\right| \boldsymbol{r}^{\prime}\right\rangle & \equiv \gamma\left(\boldsymbol{r}, \boldsymbol{r}^{\prime}\right)=\sum_{i}\langle\boldsymbol{r} \mid i\rangle\left\langle i \mid \boldsymbol{r}^{\prime}\right\rangle=\sum_{i} b_{i}(\boldsymbol{r}) b_{i}^{*}\left(\boldsymbol{r}^{\prime}\right),
\end{aligned}
$$

separates the $P$-component, $|\Delta \rho\rangle_{P}$, of a general density displacement vector $|\Delta \rho\rangle$ :

$$
\begin{aligned}
\hat{\mathrm{P}}_{P}|\Delta \rho\rangle & \equiv|\Delta \rho\rangle_{P}=\sum_{i}|i\rangle\langle i \mid \Delta \rho\rangle \equiv \sum_{i}|i\rangle \Delta \rho_{i}, \\
\Delta \rho_{i} & =\int b_{i}^{*}(\boldsymbol{r}) \Delta \rho(\boldsymbol{r}) \mathrm{d} \boldsymbol{r}, \quad \text { or } \\
\Delta \rho_{P}(\boldsymbol{r}) & =\langle\boldsymbol{r} \mid \Delta \rho\rangle_{P}=\int \gamma\left(\boldsymbol{r}, \boldsymbol{r}^{\prime}\right) \Delta \rho\left(\boldsymbol{r}^{\prime}\right) \mathrm{d} \boldsymbol{r}^{\prime} \\
& =\sum_{i} b_{i}(\boldsymbol{r}) \Delta \rho_{i} .
\end{aligned}
$$

Next, let us express a general displacement of the system density, $\rho(\boldsymbol{r}) \equiv N p(\boldsymbol{r})$, in terms of the associated changes in its normalization, $\Delta N$, and the shape" (probability) factor, $\Delta p(\boldsymbol{r})$ :

$$
\begin{gathered}
|\Delta \rho\rangle=N|\Delta p\rangle+|p\rangle \Delta N \equiv|\Delta \rho\rangle_{N}+|\Delta \rho\rangle_{C T} \quad \text { or } \\
\Delta \rho(\boldsymbol{r})=N \Delta p(\boldsymbol{r})+p(\boldsymbol{r}) \Delta N \equiv \Delta \rho_{N}(\boldsymbol{r})+\Delta \rho_{C T}(\boldsymbol{r}),
\end{gathered}
$$

where $\int p(\boldsymbol{r}) \mathrm{d} \boldsymbol{r}=1, N=\int \rho(\boldsymbol{r}) \mathrm{d} \boldsymbol{r} \equiv N[\rho]$, and hence: $\int \Delta p(\boldsymbol{r}) \mathrm{d} \boldsymbol{r}=0, \int \Delta \rho(\boldsymbol{r}) \mathrm{d} \boldsymbol{r}=\Delta N$. It follows from the preceding equation that the closed system polarization part $\Delta \rho_{N}(\boldsymbol{r})$, which integrates to zero, is purely polarizational: $\hat{\mathrm{P}}_{P}|\Delta \rho\rangle_{N}=|\Delta \rho\rangle_{N}$ (see figure 1). The $\Delta N$ normalized CT-component $\Delta \rho_{C T}(\boldsymbol{r})$, however, must contain the non-vanishing $P$ - and CT-displacements, since the added (or removed) $\Delta N$ elec- 


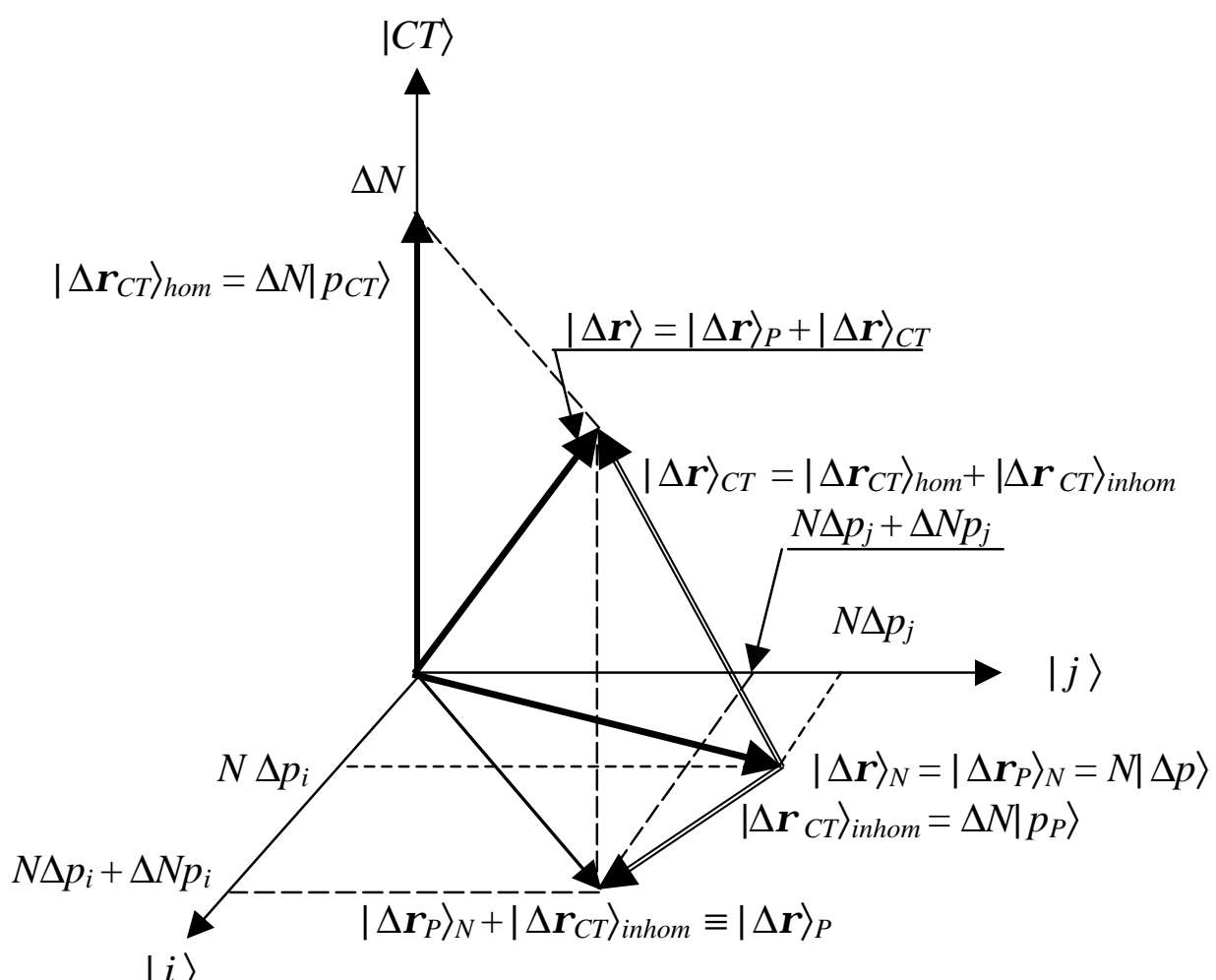

Figure 1. The density displacement vectors $|\Delta \rho\rangle_{N}$ and $|\Delta \rho\rangle$, of the closed and open molecular systems, respectively, and their partitions into the $P$ - and CT-components in the Hilbert space spanned by the two polarizational modes $\{|i\rangle,|j\rangle\}$ and the homogeneous CTmode $|\mathrm{CT}\rangle$.

trons are distributed in accordance with the initial probability factor. Indeed, this density change can be viewed as a combination of the homogeneous density displacement $\Delta \rho_{C T}{ }^{\text {hom }}$, such that $\Delta \rho_{C T}{ }^{\text {hom }} V=$ $\Delta N \neq 0$, where $V$ denotes the (finite) molecular volume, which does not bias any local volume element in the molecule, and the remaining, polarizational part $\Delta \rho_{C T}^{\text {inhom }}(\boldsymbol{r})$, which integrates to zero:

$$
\Delta \rho_{C T}(\boldsymbol{r})=\Delta \rho_{C T}^{\text {hom }}(\boldsymbol{r})+\Delta \rho_{C T}^{\text {inhom }}(\boldsymbol{r}) .
$$

These two components, shown in figure 1, are represented by the corresponding vectors in the molecular Hilbert space:

$$
\begin{gathered}
\hat{\mathrm{P}}_{P}|\Delta \rho\rangle_{C T}=\Delta N \hat{\mathrm{P}}_{P}|p\rangle \equiv \Delta N\left|p_{P}\right\rangle=\left|\Delta \rho_{C T}\right\rangle_{\text {inhom }} \\
\hat{\mathrm{P}}_{C T}|\Delta \rho\rangle_{C T}=\Delta N \hat{\mathrm{P}}_{C T}|p\rangle \equiv \Delta N\left|p_{C T}\right\rangle=\left|\Delta \rho_{C T}\right\rangle_{\text {hom }}
\end{gathered}
$$

The sum of the closed-system polarization and the inhomogeneous CT-component then defines the overall polarization in the open molecular system:

$$
\begin{gathered}
|\Delta \rho\rangle_{P}=\hat{\mathrm{P}}_{P}|\Delta \rho\rangle=|\Delta \rho\rangle_{N}+\left|\Delta \rho_{C T}\right\rangle_{\text {inhom }} \text { or } \\
\Delta \rho_{P}(\boldsymbol{r})=\left\langle\boldsymbol{r}\left|\hat{\mathrm{P}}_{P}\right| \Delta \rho\right\rangle=\Delta \rho_{N}(\boldsymbol{r})+\Delta \rho_{C T}^{\text {inhom }}(\boldsymbol{r}) .
\end{gathered}
$$

In the density displacement Hilbert space the chemical softness and hardness operators, $\hat{\sigma}$ and $\hat{\eta}$, respectively, can be similarly defined in terms of their common (orthonormal) eigenvectors $|A|=\{|\alpha\rangle\}$, $\langle\alpha \mid \beta\rangle=\delta_{\alpha, \beta}$, or their position representations $\boldsymbol{c}(\boldsymbol{r})=$ $\langle\boldsymbol{r} \mid \boldsymbol{A}\rangle=\left\{c_{\alpha}(\boldsymbol{r})=\langle\boldsymbol{r} \mid \alpha\rangle\right\}$, and the corresponding eigenvalues, $=\left\{\eta_{\alpha}\right\}$ and $=\left\{\sigma_{\alpha}=1 / \eta_{\alpha}\right\}$ :

$$
\hat{\eta}|\alpha\rangle=\eta_{\alpha}|\alpha\rangle, \quad \hat{\sigma}|\alpha\rangle=\sigma_{\alpha}|\alpha\rangle, \alpha=1,2, \ldots,
$$

or in the electron position representation

$$
\begin{aligned}
& \int\left\langle\boldsymbol{r}|\hat{\eta}| \boldsymbol{r}^{\prime}\right\rangle\left\langle\boldsymbol{r}^{\prime} \mid \alpha\right\rangle \mathrm{d} \boldsymbol{r}^{\prime} \equiv \int \eta\left(\boldsymbol{r}, \boldsymbol{r}^{\prime}\right) c_{\alpha}\left(\boldsymbol{r}^{\prime}\right) \mathrm{d} \boldsymbol{r}^{\prime}=\eta_{\alpha} c_{\alpha}(\boldsymbol{r}), \\
& \int\left\langle\boldsymbol{r}|\hat{\sigma}| \boldsymbol{r}^{\prime}\right\rangle\left\langle\boldsymbol{r}^{\prime} \mid \alpha\right\rangle \mathrm{d} \boldsymbol{r}^{\prime} \equiv \int \sigma\left(\boldsymbol{r}, \boldsymbol{r}^{\prime}\right) c_{\alpha}\left(\boldsymbol{r}^{\prime}\right) \mathrm{d} \boldsymbol{r}^{\prime}=\sigma_{\alpha} c_{\alpha}(\boldsymbol{r}), \\
& \langle\alpha \mid \beta\rangle=\int\langle\alpha \mid \boldsymbol{r}\rangle\langle\boldsymbol{r} \mid \beta\rangle \mathrm{d} \boldsymbol{r} \equiv \int c_{\alpha}^{*}(\boldsymbol{r}) c_{\beta}(\boldsymbol{r}) \mathrm{d} \boldsymbol{r}=\delta_{\alpha, \beta} .(10)
\end{aligned}
$$


Here, the eigenvalues and represent the system principal hardnesses and softnesses, respectively. ${ }^{3,4}$

The resulting spectral resolutions of these two operators then read:

$$
\begin{aligned}
\hat{\eta} & =\sum_{\alpha}|\alpha\rangle \eta_{\alpha}\langle\alpha|, \quad \text { or } \\
\eta\left(\boldsymbol{r}, \boldsymbol{r}^{\prime}\right) & =\left\langle\boldsymbol{r}|\hat{\eta}| \boldsymbol{r}^{\prime}\right\rangle=\sum_{\alpha} c_{\alpha}(\boldsymbol{r}) \eta_{\alpha} c_{\alpha}^{*}\left(\boldsymbol{r}^{\prime}\right) ; \\
\hat{\sigma} & =\sum_{\alpha}|\alpha\rangle \sigma_{\alpha}\langle\alpha|, \quad \text { or } \\
\sigma\left(\boldsymbol{r}, \boldsymbol{r}^{\prime}\right) & =\left\langle\boldsymbol{r}|\hat{\sigma}| \boldsymbol{r}^{\prime}\right\rangle=\sum_{\alpha} c_{\alpha}(\boldsymbol{r}) \sigma_{\alpha} c_{\alpha}^{*}\left(\boldsymbol{r}^{\prime}\right) .
\end{aligned}
$$

These independent, collective density displacement modes, called the density normal modes (DNM) $)^{4,5}$ span the complete vector space, capable of describing general displacements of the electron density, including both the $\mathrm{CT}$ - and $P$-components:

$$
\begin{gathered}
\hat{\mathrm{P}}|\Delta \rho\rangle \equiv \sum_{\alpha}|\alpha\rangle\langle\alpha \mid \Delta \rho\rangle \equiv \sum_{\alpha}|\alpha\rangle \Delta \rho_{\alpha}=|\Delta \rho\rangle, \\
\hat{\mathrm{P}} \equiv \sum_{\alpha}|\alpha\rangle\langle\alpha|=1,\left\langle\boldsymbol{r}|\hat{\mathrm{P}}| \boldsymbol{r}^{\prime}\right\rangle=\delta\left(\boldsymbol{r}^{\prime}-\boldsymbol{r}\right), \\
\int\left\langle\boldsymbol{r}|\hat{\mathrm{P}}| \boldsymbol{r}^{\prime}\right\rangle\left\langle\boldsymbol{r}^{\prime} \mid \Delta \rho\right\rangle \mathrm{d} \boldsymbol{r}^{\prime}=\int \delta\left(\boldsymbol{r}^{\prime}-\boldsymbol{r}\right) \Delta \rho\left(\boldsymbol{r}^{\prime}\right) \mathrm{d} \boldsymbol{r}^{\prime}=\Delta \rho(\boldsymbol{r}) \\
=\sum_{\alpha} c_{\alpha}(\boldsymbol{r}) \Delta \rho_{\alpha}, \Delta \rho_{\alpha}=\int c_{\alpha}^{*}(\boldsymbol{r}) \Delta \rho(\boldsymbol{r}) \mathrm{d} \boldsymbol{r} .
\end{gathered}
$$

Therefore the $C T$-projector is given by the difference

$$
\hat{\mathrm{P}}_{C T}=\hat{\mathrm{P}}-\hat{\mathrm{P}}_{P}=\sum_{\alpha}|\alpha\rangle\left\langle\alpha\left|-\sum_{i}\right| i\right\rangle\langle i|=1-\hat{\mathrm{P}}_{P} .
$$

It projects out the homogeneous CT component [see $(8 c)]$ of a general density displacement

$$
\begin{aligned}
\hat{\mathrm{P}}_{C T}|\Delta \rho\rangle & \equiv\left|\Delta \rho_{C T}\right\rangle_{h o m}=|\Delta \rho\rangle-|\Delta \rho\rangle_{P}=\left|p_{C T}\right\rangle \Delta N \quad \text { or } \\
\Delta \rho_{C T}^{\text {hom }}(\boldsymbol{r}) & =\left\langle\boldsymbol{r} \mid \Delta \rho_{C T}\right\rangle_{h o m}=\Delta \rho(\boldsymbol{r})-\Delta \rho_{P}(\boldsymbol{r}) \\
& =p_{C T} \Delta N,
\end{aligned}
$$

where $p_{C T}=1 / V=\left\langle\boldsymbol{r} \mid p_{C T}\right\rangle$.

In the previous analysis ${ }^{11}$ the complete vector space capable of representing any displacement of the molecular electron density was constructed by supplementing the closed system $P$-modes of (4) with the homogeneous CT-mode, capable of repre- senting changes in the density normalization. For this additional mode $a(\boldsymbol{r})=\langle\boldsymbol{r} \mid \mathrm{CT}\rangle$ to be orthogonal to all polarizational functions $\boldsymbol{b}(\boldsymbol{r})$ [see (2)] it has to be constant throughout space: $a(\boldsymbol{r}) \equiv V^{-1 / 2}=$ const. Alternatively, to avoid problems with an infinite molecular volume, one can select $V$ locally, as the volume $V \equiv V_{s}(\boldsymbol{r})=(4 / 3) \pi r_{s}(\mathbf{r})^{3}$ of the Wigner-Seitz sphere of the homogeneous electron gas, with the density dependent radius $r_{s}(\boldsymbol{r})=\rho^{-1}(\boldsymbol{r})$, so that $V_{s}(\boldsymbol{r})$ by definition contains a single electron, $V_{s}(\boldsymbol{r}) \rho(\boldsymbol{r})=1$. In this way one can interpret the constant (renormalized) CT-mode $|\boldsymbol{C T}\rangle$ as

$$
\bar{a}(\boldsymbol{r})=\langle\boldsymbol{r} \mid \boldsymbol{C} \boldsymbol{T}\rangle \equiv V_{s}(\boldsymbol{r}) \rho(\boldsymbol{r})=1 .
$$

These vectors explicitly define the corresponding CT-projectors:

$$
\begin{aligned}
& \overline{\hat{\mathrm{P}}}_{C \boldsymbol{T}}=|\boldsymbol{C} \boldsymbol{T}\rangle\langle\boldsymbol{C} \boldsymbol{T}|, \quad\left\langle\boldsymbol{r}\left|\overline{\hat{\mathrm{P}}}_{C T}\right| \boldsymbol{r}^{\prime}\right\rangle=1 ; \\
& \hat{\mathrm{P}}_{C T}=|C T\rangle\langle C T|,\left\langle\boldsymbol{r}\left|\hat{\mathrm{P}}_{C T}\right| \boldsymbol{r}^{\prime}\right\rangle=V^{-1} .
\end{aligned}
$$

The $P$-modes then reflect the local aspect of shifts in the electron density, while the CT-mode corresponds to the homogeneous (normalization) facet of the electron distribution in the molecule. Since the two homogeneous CT-projectors are renormalized versions of each other, in what follows we shall explicitly use the $\hat{\mathrm{P}}^{C T}$ operator, which makes reference to the molecular volume. Since $\hat{\mathrm{P}}=\hat{\mathrm{P}}^{C T}+\hat{\mathrm{P}}_{P}=1$ the overall projection onto the whole $(\mathrm{CT}, P)$-vector space gives:

$$
\left\langle\boldsymbol{r}|\hat{\mathrm{P}}| \boldsymbol{r}^{\prime}\right\rangle=\delta\left(\boldsymbol{r}^{\prime}-\boldsymbol{r}\right)=V^{-1}+\gamma\left(\boldsymbol{r}, \boldsymbol{r}^{\prime}\right) .
$$

Let us again examine the result of the CT-projection of $|\Delta \rho\rangle$ :

$$
\begin{aligned}
\left\langle\boldsymbol{r}\left|\hat{\mathrm{P}}_{C T}\right| \Delta \rho\right\rangle & =\Delta N\left\langle\boldsymbol{r}\left|\hat{\mathrm{P}}_{C T}\right| p\right\rangle=\Delta N\left\langle\boldsymbol{r} \mid p_{C T}\right\rangle \\
& \equiv \Delta N p_{C T}(\boldsymbol{r})=\int\left\langle\boldsymbol{r}\left|\hat{\mathrm{P}}_{C T}\right| \boldsymbol{r}^{\prime}\right\rangle\left\langle\boldsymbol{r}^{\prime} \mid \Delta \rho\right\rangle \mathrm{d} \boldsymbol{r}^{\prime} \\
& =V^{-1} \int \Delta \rho\left(\boldsymbol{r}^{\prime}\right) \mathrm{d} \boldsymbol{r}^{\prime}=\Delta N / V \equiv \Delta \rho_{C T}^{\text {hom }},
\end{aligned}
$$

Thus the additional CT-mode indeed describes the homogeneous CT-displacement of the system electronic density (see figure 1), which does not bias any location in space.

The $P$-projection of the density displacement vector similarly gives [see (7) and (8d)]: 


$$
\begin{aligned}
& \left\langle\boldsymbol{r}\left|\hat{\mathrm{P}}_{P}\right| \Delta \rho\right\rangle=N\left\langle\boldsymbol{r}\left|\hat{\mathrm{P}}_{P}\right| \Delta p\right\rangle+\Delta N\left\langle\boldsymbol{r}\left|\hat{\mathrm{P}}_{P}\right| p\right\rangle \\
& \equiv N\langle\boldsymbol{r} \mid \Delta p\rangle+\Delta N\left\langle\boldsymbol{r} \mid p_{P}\right\rangle \equiv N \Delta p(\boldsymbol{r})+\Delta N p_{P}(\boldsymbol{r}) \\
& =\int\left\langle\boldsymbol{r}\left|\hat{\mathrm{P}}_{P}\right| \boldsymbol{r}^{\prime}\right\rangle\left\langle\boldsymbol{r}^{\prime} \mid \Delta \rho\right\rangle=\int\left[\delta\left(\boldsymbol{r}^{\prime}-\boldsymbol{r}\right)-V^{-1}\right] \Delta \rho\left(\boldsymbol{r}^{\prime}\right) \mathrm{d} \boldsymbol{r}^{\prime} \\
& \equiv \Delta \rho_{P}(\boldsymbol{r})=\Delta \rho(\boldsymbol{r})-\Delta N / V \\
& =N \Delta p(\boldsymbol{r})+\Delta N\left[p(\boldsymbol{r})-V^{-1}\right] \equiv \Delta \rho_{N}(\boldsymbol{r})+\Delta \rho_{C T}^{\text {inhom }}(\boldsymbol{r}) .
\end{aligned}
$$

Rewriting $\Delta \rho_{P}(\boldsymbol{r})$ in terms of the $P$-modes $\boldsymbol{b}(\boldsymbol{r})$ also gives (see figure 1):

$$
\begin{aligned}
& \left\langle\boldsymbol{r}\left|\hat{\mathrm{P}}_{P}\right| \Delta \rho\right\rangle=\sum_{i} b_{i}(\boldsymbol{r})\left[\int b_{i} *\left(\boldsymbol{r}^{\prime}\right) \Delta \rho\left(\boldsymbol{r}^{\prime}\right) \mathrm{d} \boldsymbol{r}^{\prime}\right] \equiv \sum_{i} b_{i}(\boldsymbol{r}) \Delta \boldsymbol{\rho}_{i} \\
& =\sum_{i} b_{i}(\boldsymbol{r})\left\{N\left[\int b_{i} *\left(\boldsymbol{r}^{\prime}\right) \Delta p\left(\boldsymbol{r}^{\prime}\right) \mathrm{d} \boldsymbol{r}^{\prime}\right]+\Delta N\left[\int b_{i}^{*}\left(\boldsymbol{r}^{\prime}\right) p\left(\boldsymbol{r}^{\prime}\right) \mathrm{d} \boldsymbol{r}^{\prime}\right]\right\} \\
& \equiv \sum_{i} b_{i}(\boldsymbol{r})\left(N \Delta p_{i}+\Delta N p_{i}\right) .
\end{aligned}
$$

Therefore, the homogeneous CT-component, $\Delta \rho_{C T}^{h o m}(\boldsymbol{r}) \equiv\left\langle\boldsymbol{r} \mid \Delta \rho_{C T}\right\rangle_{h o m}$, of the open system displacement in the electron density indeed corresponds to the uniform distribution of $\Delta N$ electrons in the whole (finite) molecular volume, while its overall $P$-component $\Delta \rho_{P}(\boldsymbol{r}) \equiv\langle\boldsymbol{r} \mid \Delta \rho\rangle_{P}$ now contains the closed-system polarization density, $\Delta \rho_{N}(\boldsymbol{r}) \equiv N \Delta p(\boldsymbol{r})$ $\equiv\left\langle\boldsymbol{r} \mid \Delta \rho_{P}\right\rangle_{N}$, and the inhomogeneous part of the CTcomponent, $\Delta \rho_{C T}^{\text {inhom }}(\boldsymbol{r}) \equiv\left\langle\boldsymbol{r} \mid \Delta \rho_{C T}\right\rangle_{\text {inhom }}$ (see figure 1). As also shown in the figure the two CT-related vectors define the overall density displacement vector

$$
|\Delta \rho\rangle_{C T}=\left|\Delta \rho_{C T}\right\rangle_{h o m}+\left|\Delta \rho_{C T}\right\rangle_{\text {inhom }}=\Delta N|p\rangle \text {. }
$$

The above inhomogeneous CT-component should not be confused with the CT-induced polarization, which represents the second-order effect involving the product of $\Delta N$ and $|\Delta p\rangle$.

As indicated in (8c) the above partitioning of the density CT-displacement into its homogeneous and inhomegeneous components results from the division of the probability (shape-factor) vector:

$$
|p\rangle=\left(\hat{\mathrm{P}}_{P}+\hat{\mathrm{P}}_{C T}\right)|p\rangle \equiv\left|p_{P}\right\rangle+\left|p_{C T}\right\rangle .
$$

It then follows from (19) and (20) that

$$
\begin{gathered}
\left\langle\boldsymbol{r} \mid p_{C T}\right\rangle=p_{C T}(\boldsymbol{r})=V^{-1} \quad \text { and } \\
\left\langle\boldsymbol{r} \mid p_{P}\right\rangle=p_{P}(\boldsymbol{r})=p(\boldsymbol{r})-V^{-1} .
\end{gathered}
$$

The resultant polarization vector of the open molecular system [(8d), (20), (21)] thus includes the inhomogeneous $\Delta N$-dependent term, representing the pseudo-polarization relative to the homogeneous background of the CT-mode, which results from distributing $\Delta N$ electrons among local volume elements in proportion to the local shape factor $p(\boldsymbol{r})$. This extra displacement is explicitly shown in figure 1. It follows from (21) that the closed system term $\langle\boldsymbol{r} \mid \Delta \rho\rangle_{N}=N \sum_{i} b_{i}(\boldsymbol{r}) \Delta p_{i}=N \Delta p(\boldsymbol{r})$, while the inhomoheneous part of of the CT-displacement of the electron density $\left\langle\boldsymbol{r} \mid \Delta \rho_{C T}\right\rangle_{\text {inhom }}=\Delta N p_{P}(\boldsymbol{r})=\Delta N \sum_{i} b_{i}(\boldsymbol{r}) p_{i}$.

\section{Density-potential relations}

Let us summarize the relations linking the shifts $|\Delta \rho\rangle$ in the system electron density $\Delta \rho(r)$ and the corresponding vectors representing changes in the external potential, $\langle\boldsymbol{r} \mid \Delta \mathrm{v}\rangle=\Delta v(\boldsymbol{r})$, and the relative potential, $\langle\boldsymbol{r} \mid \Delta u\rangle=\Delta u(\boldsymbol{r})=\Delta v(\boldsymbol{r})-\Delta \mu(\boldsymbol{r})$, where $\Delta \mu(\boldsymbol{r})=$ $\langle\boldsymbol{r} \mid \Delta \mu\rangle$ stands for the displacement in the system local chemical potential of electrons. The latter is equalized for the ground-state (equilibrium) density at the global chemical potential level $\mu[N, v],{ }^{2,4-8}$

$\mu(\boldsymbol{r})=\frac{\delta E_{v}[\rho]}{\delta \rho(\boldsymbol{r})}=v(r)+\frac{\delta F[\rho]}{\delta \rho(\boldsymbol{r})}=\mu[N, v]=\left(\frac{\partial E[N, v]}{\partial N}\right)_{v}$

or

$u(\boldsymbol{r}) \equiv v(\boldsymbol{r})-\mu=-\frac{\delta F[\rho]}{\delta \rho(\boldsymbol{r})}$,

where, the universal ( $v$-independent) functional $F[\rho]=T_{e}[\rho]+V_{e e}[\rho]$ of the density functional for the system electronic energy,

$$
E_{v}[\rho]=\int \rho(\boldsymbol{r}) v(\boldsymbol{r}) \mathrm{d} \boldsymbol{r}+F[\rho],
$$

generates the sum of the electronic kinetic $\left(T_{e}\right)$ and repulsion $\left(V_{e e}\right)$ energies. The chemical potential (electronegativity) equalization of (25a) is implied by the Hohenberg-Kohn [1] Euler equation derived from the $\mathrm{N}$-constrained minimum principle for the system electronic energy:

$$
\delta\left\{E_{v}[\rho]-\mu[N, v] N[\rho]\right\}=0 .
$$

Equation (25a) implies the following expression for the linear response in the relative external potential:

$$
\Delta u(\boldsymbol{r}) \equiv \Delta v(\boldsymbol{r})-\Delta \mu=-\Delta\left(\frac{\delta F[\rho]}{\delta \rho(\boldsymbol{r})}\right)
$$




$$
\begin{aligned}
& =-\int \Delta \rho\left(\boldsymbol{r}^{\prime}\right) \frac{\delta^{2} F[\rho]}{\delta \rho\left(\boldsymbol{r}^{\prime}\right) \delta \rho(\boldsymbol{r})} \mathrm{d} \boldsymbol{r}^{\prime} \\
& \equiv-\int \Delta \rho\left(\boldsymbol{r}^{\prime}\right) \eta\left(\boldsymbol{r}^{\prime}, \boldsymbol{r}\right) \mathrm{d} \boldsymbol{r}^{\prime},
\end{aligned}
$$

where the chemical hardness kernel,

$$
\eta\left(\boldsymbol{r}^{\prime}, \boldsymbol{r}\right)=\left\langle\boldsymbol{r}^{\prime}|\hat{\eta}| \boldsymbol{r}\right\rangle=-\frac{\delta u(\boldsymbol{r})}{\delta \rho\left(\boldsymbol{r}^{\prime}\right)} \equiv-\frac{\langle\delta u \mid \boldsymbol{r}\rangle}{\left\langle\boldsymbol{r}^{\prime} \mid \delta \rho\right\rangle},
$$

represents the chemical hardness operator $\hat{\eta}=$ $-\langle\delta u|/| \delta \rho\rangle$. Equation (27) can be written in the equivalent vector form:

$$
|\Delta u\rangle=|\Delta v\rangle-|\Delta \mu\rangle=-\hat{\eta}|\Delta \rho\rangle .
$$

The inverse relation,

$$
|\Delta \rho\rangle=-\hat{\sigma}|\Delta u\rangle,
$$

is obtained by acting on both sides of (29) with the chemical softness operator $\hat{\sigma}=\hat{\eta}^{-1}=-\langle\delta \rho|/| \delta u\rangle$, which gives rise to the softness kernel

$\sigma\left(\boldsymbol{r}^{\prime}, \boldsymbol{r}\right)=\eta^{-1}\left(\boldsymbol{r}^{\prime}, \boldsymbol{r}\right)=\left\langle\boldsymbol{r}^{\prime}|\hat{\sigma}| \boldsymbol{r}\right\rangle=-\frac{\delta \rho(\boldsymbol{r})}{\delta u\left(\boldsymbol{r}^{\prime}\right)} \equiv-\frac{\langle\delta \rho \mid \boldsymbol{r}\rangle}{\left\langle\boldsymbol{r}^{\prime} \mid \delta u\right\rangle}$.

These inverse kernels satisfy the functional reciprocity relation:

$$
\begin{aligned}
\int \sigma\left(\boldsymbol{r}, \boldsymbol{r}^{\prime \prime}\right) \eta\left(\boldsymbol{r}^{\prime \prime}, \boldsymbol{r}^{\prime}\right) \mathrm{d} \boldsymbol{r}^{\prime \prime} & =\int \frac{\delta \rho\left(\boldsymbol{r}^{\prime \prime}\right)}{\delta u(\boldsymbol{r})} \frac{\delta u\left(\boldsymbol{r}^{\prime}\right)}{\delta \rho\left(\boldsymbol{r}^{\prime \prime}\right)} \mathrm{d} \boldsymbol{r}^{\prime \prime} \\
& =\frac{\delta u\left(\boldsymbol{r}^{\prime}\right)}{\delta u(\boldsymbol{r})}=\delta\left(\boldsymbol{r}^{\prime}-\boldsymbol{r}\right) .
\end{aligned}
$$

In the externally closed molecular system, for which the density displacement $\Delta \rho_{N}(\boldsymbol{r})=\langle\boldsymbol{r} \mid \Delta \rho\rangle_{N}=$ $N \Delta p(\boldsymbol{r})$ [see (3)],

$$
{ }_{N}\langle\Delta \rho|=N\langle\Delta p|=\langle\Delta v| \hat{\beta}=\langle\Delta v| \frac{{ }_{N}\langle\delta \rho|}{|\delta v\rangle} .
$$

In the position representation this operator equation in the DDM Hilbert space assumes the familiar form:

$$
\Delta \rho_{N}(\boldsymbol{r})={ }_{N}\langle\Delta \rho \mid \boldsymbol{r}\rangle=N \Delta p(\boldsymbol{r})=\int\left\langle\Delta v \mid \boldsymbol{r}^{\prime}\right\rangle \frac{{ }_{N}\langle\delta \rho \mid \boldsymbol{r}\rangle}{\left\langle\boldsymbol{r}^{\prime} \mid \delta v\right\rangle} \mathrm{d} \boldsymbol{r}^{\prime}
$$

$$
=\int \Delta v\left(\boldsymbol{r}^{\prime}\right) \beta\left(\boldsymbol{r}^{\prime}, \boldsymbol{r}\right) \mathrm{d} \boldsymbol{r}^{\prime} .
$$

The corresponding inverse relations are:

$$
\langle\Delta v|={ }_{N}\langle\Delta \rho| \hat{\beta}^{-1}=N\langle\Delta p| \frac{\langle\delta v|}{|\delta \rho\rangle_{N}},
$$

or in the position representation:

$$
\begin{aligned}
\langle\Delta v \mid \boldsymbol{r}\rangle & =\Delta v(\boldsymbol{r})=N \int\left\langle\Delta p \mid \boldsymbol{r}^{\prime}\right\rangle \frac{\langle\delta v \mid \boldsymbol{r}\rangle}{\left\langle\boldsymbol{r}^{\prime} \mid \delta \rho\right\rangle_{N}} \mathrm{~d} \boldsymbol{r}^{\prime} \\
& =N \int \Delta p\left(\boldsymbol{r}^{\prime}\right) \beta^{-1}\left(\boldsymbol{r}^{\prime}, \boldsymbol{r}\right) \mathrm{d} \boldsymbol{r}^{\prime} .
\end{aligned}
$$

The so-called internal hardness operator, ${ }^{11}$

$$
\hat{\eta}^{\text {int }} \equiv-\hat{\beta}^{-1}=-\sum_{i}|i\rangle \beta_{i}^{-1}\langle i|=-\frac{\langle\delta v|}{|\delta \rho\rangle_{N}},
$$

defines the internal hardness kernel of the closed molecular system:

$$
\begin{aligned}
\eta^{i n t}\left(\boldsymbol{r}, \boldsymbol{r}^{\prime}\right) & =-\frac{\left\langle\delta v \mid \boldsymbol{r}^{\prime}\right\rangle}{\langle\boldsymbol{r} \mid \delta \rho\rangle_{N}}=-\beta^{-1}\left(\boldsymbol{r}, \boldsymbol{r}^{\prime}\right) \\
& =-\left(\frac{\partial v\left(\boldsymbol{r}^{\prime}\right)}{\partial \rho(\boldsymbol{r})}\right)_{N} \equiv-\left\langle\boldsymbol{r}\left|\hat{\beta}^{-1}\right| \boldsymbol{r}^{\prime}\right\rangle .
\end{aligned}
$$

The negative density LR kernel of (3) similarly defines the internal softness kernel of the closed molecular system, the position representation of the corresponding operator

$$
\begin{aligned}
\hat{\boldsymbol{\sigma}}^{\text {int }} & \equiv-\hat{\beta}=-\sum_{i}|i\rangle \beta_{i}\langle i|=-\frac{{ }_{N}\langle\delta \rho|}{|\delta v\rangle}, \\
\sigma^{i n t}\left(\boldsymbol{r}, \boldsymbol{r}^{\prime}\right) & =-\frac{{ }_{N}\left\langle\delta \rho \mid \boldsymbol{r}^{\prime}\right\rangle}{\langle\boldsymbol{r} \mid \delta v\rangle}=-\beta\left(\boldsymbol{r}, \boldsymbol{r}^{\prime}\right) \\
& =-\left(\frac{\partial \rho\left(\boldsymbol{r}^{\prime}\right)}{\partial v(\boldsymbol{r})}\right)_{N} \equiv-\left\langle\boldsymbol{r}|\hat{\mathrm{a}}| \boldsymbol{r}^{\prime}\right\rangle
\end{aligned}
$$

The operator equations linking the density and the external potential vectors of the open molecular system are given in (29) and (30). The linear responses in the system relative potential due to a given perturbation in the electron distribution are defined by the hardness operator/kernel:

$$
\begin{aligned}
\langle\Delta u| & =\langle\Delta v|-\langle\Delta \mu|=-\langle\Delta \rho| \hat{\eta} \\
& =-N\langle\Delta p| \hat{\eta}-\Delta N\langle p| \hat{\eta},
\end{aligned}
$$




$$
\begin{aligned}
\langle\Delta u \mid \boldsymbol{r}\rangle & =\Delta u(\boldsymbol{r})=\Delta v(\boldsymbol{r})-\Delta \mu \\
& =[\Delta u(\boldsymbol{r})]_{\mu}+[\Delta u(\boldsymbol{r})]_{v} \\
& =-\int\left\langle\Delta \rho \mid \boldsymbol{r}^{\prime}\right\rangle\left\langle\boldsymbol{r}^{\prime}|\hat{\eta}| \boldsymbol{r}\right\rangle \mathrm{d} \boldsymbol{r}^{\prime}=-\int \Delta \rho\left(\boldsymbol{r}^{\prime}\right) \eta\left(\boldsymbol{r}^{\prime}, \boldsymbol{r}\right) \mathrm{d} \boldsymbol{r}^{\prime} \\
& =-\int\left[N \Delta p\left(\boldsymbol{r}^{\prime}\right)+\Delta N p\left(\boldsymbol{r}^{\prime}\right)\right] \eta\left(\boldsymbol{r}^{\prime}, \boldsymbol{r}\right) \mathrm{d} \boldsymbol{r}^{\prime} \\
& =-\int\left\{\left[\Delta \rho\left(\boldsymbol{r}^{\prime}\right)\right]_{N}+\left[\Delta \rho\left(\boldsymbol{r}^{\prime}\right)\right]_{p}\right\} \eta\left(\boldsymbol{r}^{\prime}, \boldsymbol{r}\right) \mathrm{d} \boldsymbol{r}^{\prime} .
\end{aligned}
$$

The corresponding inverse relations linking perturbations in the relative potential with the equilibrium linear responses of the system electron density are determined by the system softness operator/kernel:

$$
\begin{aligned}
\langle\Delta \rho|= & N\langle\Delta p|-\Delta N\langle p|=-\langle\Delta u| \hat{\sigma} \\
= & -\langle\Delta v| \hat{\sigma}+\langle\Delta \mu| \hat{\mathrm{o}}, \\
\langle\Delta \rho \mid \boldsymbol{r}\rangle & =\Delta \rho(\boldsymbol{r})=N \Delta p(\boldsymbol{r})+\Delta N p(\boldsymbol{r}) \\
& =[\Delta \rho(\boldsymbol{r})]_{N}+[\Delta \rho(\boldsymbol{r})]_{p}=-\int\left\langle\Delta u \mid \boldsymbol{r}^{\prime}\right\rangle\left\langle\boldsymbol{r}^{\prime}|\hat{\sigma}| \boldsymbol{r}\right\rangle \mathrm{d} \boldsymbol{r}^{\prime} \\
& =-\int \Delta u\left(\boldsymbol{r}^{\prime}\right) \sigma\left(\boldsymbol{r}^{\prime}, \boldsymbol{r}\right) \mathrm{d} \boldsymbol{r}^{\prime} \\
& =\int\left[\Delta \mu-\Delta v\left(\boldsymbol{r}^{\prime}\right)\right] \sigma\left(\boldsymbol{r}^{\prime}, \boldsymbol{r}\right) \mathrm{d} \boldsymbol{r}^{\prime} \\
& =-\int\left\{\left[\Delta u\left(\boldsymbol{r}^{\prime}\right)\right]_{\mu}+\left[\Delta u\left(\boldsymbol{r}^{\prime}\right)\right]_{v}\right\} \sigma\left(\boldsymbol{r}^{\prime}, \boldsymbol{r}\right) \mathrm{d} \boldsymbol{r}^{\prime} .
\end{aligned}
$$

It follows from (42) that the hardness kernel of (28) can be alternatively expressed by the following partial derivatives involving the external and chemical potentials:

$$
\begin{aligned}
\eta\left(\boldsymbol{r}^{\prime}, \boldsymbol{r}\right) & =-\left(\frac{\partial u(\boldsymbol{r})}{\partial \rho\left(\boldsymbol{r}^{\prime}\right)}\right)_{N}=-\left(\frac{\partial v(\boldsymbol{r})}{\partial \rho\left(\boldsymbol{r}^{\prime}\right)}\right)_{N}+\left(\frac{\partial \mu}{\partial \rho\left(\boldsymbol{r}^{\prime}\right)}\right)_{N} \\
& =-\frac{\delta u(\boldsymbol{r})}{N \delta p\left(\boldsymbol{r}^{\prime}\right)}=-\left(\frac{\partial u(\boldsymbol{r})}{\partial \rho\left(\boldsymbol{r}^{\prime}\right)}\right)_{P} \\
& =-\left(\frac{\partial v(\boldsymbol{r})}{\partial \rho\left(\boldsymbol{r}^{\prime}\right)}\right)_{P}+\left(\frac{\partial \mu}{\partial \rho\left(\boldsymbol{r}^{\prime}\right)}\right)_{p}=-\frac{\delta u(\boldsymbol{r})}{p\left(\boldsymbol{r}^{\prime}\right) \delta N}
\end{aligned}
$$

Let us first examine the internal derivatives, for the fixed $N$, in the first row of the preceding equation. The first diagonal derivative represents the internal hardness kernel of (38), given by the negative inverse of the density LR kernel. It represents the external potential response due to the local change in the density resulting from the displacements in the density shape factor, for its fixed normalization.
Therefore, the other derivative, with respect to the closed system electronic density, corresponding to the shift in the system chemical potential due to such an internal density displacement, is given by the difference

$$
\begin{aligned}
& \left(\frac{\partial \mu}{\partial \rho\left(\boldsymbol{r}^{\prime}\right)}\right)_{N}=\eta\left(\boldsymbol{r}^{\prime}, \boldsymbol{r}\right)-\eta^{i n t}\left(\boldsymbol{r}^{\prime}, \boldsymbol{r}\right) \quad \text { or } \\
& \left(\frac{\partial \mu}{\partial p\left(\boldsymbol{r}^{\prime}\right)}\right)_{N}=N\left[\eta\left(\boldsymbol{r}^{\prime}, \boldsymbol{r}\right)-\eta^{i n t}\left(\boldsymbol{r}^{\prime}, \boldsymbol{r}\right)\right] .
\end{aligned}
$$

The first partial derivative of the second row in (45a), measuring the external potential response per unit change in the overall number of electrons, for the fixed density probability factor, results from the chain-rule for implicit functionals:

$$
\begin{aligned}
-\left(\frac{\partial v(\boldsymbol{r})}{\partial \rho\left(\boldsymbol{r}^{\prime}\right)}\right)_{p} & =-\left(\frac{\partial v(\boldsymbol{r})}{p\left(\boldsymbol{r}^{\prime}\right) \partial N}\right)_{p} \\
& =\int\left(\frac{\partial \rho\left(\boldsymbol{r}^{\prime \prime}\right)}{p\left(\boldsymbol{r}^{\prime}\right) \partial N}\right)_{v}\left(\frac{\partial v(\boldsymbol{r})}{\partial \rho\left(\boldsymbol{r}^{\prime \prime}\right)}\right)_{N} \mathrm{~d} \boldsymbol{r}^{\prime \prime} \\
& =p\left(\boldsymbol{r}^{\prime}\right)^{-1} \int f\left(\boldsymbol{r}^{\prime \prime}\right) \beta^{-1}\left(\boldsymbol{r}^{\prime \prime}, \boldsymbol{r}\right) \mathrm{d} \boldsymbol{r}^{\prime \prime} \equiv \frac{\beta^{-1}(\boldsymbol{r})}{p\left(\boldsymbol{r}^{\prime}\right)},
\end{aligned}
$$

where the electronic Fukui Function (FF) ${ }^{9}$

$$
f(\boldsymbol{r})=\left(\frac{\partial \rho(\boldsymbol{r})}{\partial N}\right)_{v}=\left(\frac{\partial \mu}{\partial v(\boldsymbol{r})}\right)_{N}
$$

and $\beta^{-1}(\boldsymbol{r})$ stands for the internal local hardness, which is not equalized. Thus, from (45a),

$$
\left(\frac{\partial \mu}{\partial \rho\left(\boldsymbol{r}^{\prime}\right)}\right)_{p}=\left(\frac{\partial \mu}{p\left(\boldsymbol{r}^{\prime}\right) \partial N}\right)_{p}=\eta\left(\boldsymbol{r}^{\prime}, \boldsymbol{r}\right)-\frac{\beta^{-1}(\boldsymbol{r})}{p\left(\boldsymbol{r}^{\prime}\right)} .
$$

A similar division can be carried out for the softness kernel. It can be expressed using the functional chain-rule transformation in terms the closed system density response, and the extra term present in the open molecular systems, which involves the electronic FF and the system global softness. ${ }^{9}$ For this purpose we express the ground state density as functional of $N$ and $v, \rho=\rho[N, v]$ :

$$
\sigma\left(\boldsymbol{r}, \boldsymbol{r}^{\prime}\right)=\eta\left(\boldsymbol{r}^{\prime}, \boldsymbol{r}\right)^{-1}=-\frac{\delta \rho\left(\boldsymbol{r}^{\prime}\right)}{\delta u(\boldsymbol{r})}=-\left(\frac{\partial \rho\left(\boldsymbol{r}^{\prime}\right)}{\partial v(\boldsymbol{r})}\right)_{\mu}
$$




$$
\begin{aligned}
& =-\left(\frac{\partial \rho\left(\boldsymbol{r}^{\prime}\right)}{\partial v(\boldsymbol{r})}\right)_{N}-\left(\frac{\partial N}{\partial v(\boldsymbol{r})}\right)_{\mu}\left(\frac{\partial \rho\left(\boldsymbol{r}^{\prime}\right)}{\partial N}\right)_{v} \\
& =-\beta\left(\boldsymbol{r}, \boldsymbol{r}^{\prime}\right)+s(\boldsymbol{r}) f\left(\boldsymbol{r}^{\prime}\right)=-\beta\left(\boldsymbol{r}, \boldsymbol{r}^{\prime}\right)+f(\boldsymbol{r}) S f\left(\boldsymbol{r}^{\prime}\right) .
\end{aligned}
$$

Here the local softness

$$
\begin{aligned}
s(\boldsymbol{r}) & =\left(\frac{\partial \rho(\boldsymbol{r})}{\partial \mu}\right)_{V}=\left(\frac{\partial \rho(\boldsymbol{r})}{\partial N}\right)_{V}\left(\frac{\partial N}{\partial \mu}\right)_{V} \\
& =f(\boldsymbol{r}) S=-\left(\frac{\partial N}{\partial v(\boldsymbol{r})}\right)_{\mu}=\int \sigma\left(\boldsymbol{r}, \boldsymbol{r}^{\prime}\right) \mathrm{d} \boldsymbol{r}^{\prime},
\end{aligned}
$$

and the global softness

$$
\begin{aligned}
S & =\left(\frac{\partial N}{\partial \mu}\right)_{V}=\iint \frac{\delta \rho\left(\boldsymbol{r}^{\prime}\right)}{\delta u(\boldsymbol{r})}\left(\frac{\delta u(\boldsymbol{r})}{\delta \mu}\right)_{V} \mathrm{~d} \boldsymbol{r} \mathrm{d} \boldsymbol{r}^{\prime} \\
& =\iint \sigma\left(\boldsymbol{r}, \boldsymbol{r}^{\prime}\right) \mathrm{d} \boldsymbol{r} \mathrm{d} \boldsymbol{r}^{\prime}=\int\left(\frac{\partial \rho(\boldsymbol{r})}{\partial \mu}\right)_{V} \mathrm{~d} \boldsymbol{r} \\
& =\int s(\boldsymbol{r}) \mathrm{d} \boldsymbol{r} .
\end{aligned}
$$

It also follows from (44) that

$$
\begin{aligned}
\sigma\left(\boldsymbol{r}^{\prime}, \boldsymbol{r}\right) & =-\left(\frac{\partial \rho(\boldsymbol{r})}{\partial u\left(\boldsymbol{r}^{\prime}\right)}\right)_{\mu}=-\left(\frac{\partial \rho(\boldsymbol{r})}{\partial v\left(\boldsymbol{r}^{\prime}\right)}\right)_{\mu} \\
& =-\left(\frac{\partial \rho(\boldsymbol{r})}{\partial u\left(\boldsymbol{r}^{\prime}\right)}\right)_{V}=-\left(\frac{\partial \rho(\boldsymbol{r})}{\partial \mu\left(\boldsymbol{r}^{\prime}\right)}\right)_{V},
\end{aligned}
$$

where the non-equilibrium (non-equalized) local displacement of the system chemical potential $[\delta \mu(\boldsymbol{r})]_{v}=\int\left[\delta \rho\left(\boldsymbol{r}^{\prime}\right)\right]_{v} \eta\left(\boldsymbol{r}^{\prime}, \boldsymbol{r}\right) \mathrm{d} \boldsymbol{r}^{\prime}$. Using (8a) and (48) then gives:

$$
\begin{aligned}
\sigma\left(\boldsymbol{r}^{\prime}, \boldsymbol{r}\right) & =-N\left(\frac{\partial p(\boldsymbol{r})}{\partial v\left(\boldsymbol{r}^{\prime}\right)}\right)_{\mu}-p(\boldsymbol{r}) s\left(\boldsymbol{r}^{\prime}\right) \\
& =N\left(\frac{\partial p(\boldsymbol{r})}{\partial \mu\left(\boldsymbol{r}^{\prime}\right)}\right)_{v}-p(\boldsymbol{r}) s\left(\boldsymbol{r}^{\prime}\right)
\end{aligned}
$$

Finally, combining (35) and (41) gives an explicit expression for the chemical potential displacement:

$$
\begin{aligned}
\langle\Delta \mu| & =\langle\Delta v|-\langle\Delta u|=\langle\Delta \rho|\left(\hat{\mathrm{P}}_{P} \hat{\beta}^{-1}+\hat{\eta}\right) \\
& ={ }_{P}\langle\Delta \rho|\left(\hat{\beta}^{-1}+\hat{\eta}\right)+{ }_{C T}\langle\Delta \rho| \hat{\eta} \quad \text { or }
\end{aligned}
$$

$$
\begin{aligned}
\Delta \mu(\boldsymbol{r})= & \Delta \mu=\int \Delta \rho_{N}\left(\boldsymbol{r}^{\prime}\right)\left[\beta^{-1}\left(\boldsymbol{r}^{\prime}, \boldsymbol{r}\right)+\eta\left(\boldsymbol{r}^{\prime}, \boldsymbol{r}\right)\right] \mathrm{d} \boldsymbol{r}^{\prime} \\
& +\int \Delta \rho_{C T}\left(\boldsymbol{r}^{\prime}\right) \eta\left(\boldsymbol{r}^{\prime}, \boldsymbol{r}\right) \mathrm{d} \boldsymbol{r}^{\prime} \\
= & \int \Delta \rho\left(\boldsymbol{r}^{\prime}\right)\left[\int \gamma\left(\boldsymbol{r}^{\prime}, \boldsymbol{r}^{\prime \prime}\right) \beta^{-1}\left(\boldsymbol{r}^{\prime \prime}, \boldsymbol{r}\right) \mathrm{d} \boldsymbol{r}^{\prime \prime}+\eta\left(\boldsymbol{r}^{\prime}, \boldsymbol{r}\right)\right] \mathrm{d} \boldsymbol{r}^{\prime} \\
\equiv & \int \Delta \rho\left(\boldsymbol{r}^{\prime}\right)\left(\frac{\delta \mu(\boldsymbol{r})}{\delta \rho\left(\boldsymbol{r}^{\prime}\right)}\right) \mathrm{d} \boldsymbol{r}^{\prime},
\end{aligned}
$$

where the position representation $\gamma\left(\boldsymbol{r}, \boldsymbol{r}^{\prime}\right)$ of the $P$ projector has been defined in (6) and (18). One also identifies the first term in (44) [see also (25) and (26)] as

$$
\begin{aligned}
\langle\Delta v| & =\langle\Delta \rho| \hat{\mathrm{P}}_{P} \hat{\beta}^{-1}={ }_{P}\langle\Delta \rho| \hat{\beta}^{-1} \quad \text { or } \\
\Delta v(\boldsymbol{r}) & =\int\left[\int \Delta \rho\left(\boldsymbol{r}^{\prime}\right) \gamma\left(\boldsymbol{r}^{\prime}, \boldsymbol{r}^{\prime \prime}\right) \mathrm{d} \boldsymbol{r}^{\prime}\right] \beta^{-1}\left(\boldsymbol{r}^{\prime \prime}, \boldsymbol{r}\right) \mathrm{d} \boldsymbol{r}^{\prime \prime} \\
& \equiv \int \Delta \rho_{P}\left(\boldsymbol{r}^{\prime \prime}\right)\left(\frac{\delta v(\boldsymbol{r})}{\delta \rho\left(\boldsymbol{r}^{\prime \prime}\right)}\right)_{N} \mathrm{~d} \boldsymbol{r}^{\prime \prime} .
\end{aligned}
$$

In (45a) we have effectively partitioned the hardness kernel into the additive components representing the linear responses in the system chemical and external potentials:

$$
\begin{aligned}
\eta\left(\boldsymbol{r}^{\prime}, \boldsymbol{r}\right) & =-\frac{\delta u(\boldsymbol{r})}{\delta \rho\left(\boldsymbol{r}^{\prime}\right)}=-\frac{\langle\delta u \mid \boldsymbol{r}\rangle}{\left\langle\boldsymbol{r}^{\prime} \mid \delta \rho\right\rangle} \\
& =\left(\frac{\delta \mu(\boldsymbol{r})}{\delta \rho\left(\boldsymbol{r}^{\prime}\right)}\right)-\left(\frac{\delta v(\boldsymbol{r})}{\delta \rho\left(\boldsymbol{r}^{\prime}\right)}\right) \\
& =\frac{\langle\delta \mu \mid \boldsymbol{r}\rangle}{\left\langle\boldsymbol{r}^{\prime} \mid \delta \rho\right\rangle}-\frac{\langle\delta v \mid \boldsymbol{r}\rangle}{\left\langle\boldsymbol{r}^{\prime} \mid \delta \rho\right\rangle} .
\end{aligned}
$$

Equation (50) identifies the corresponding inverse functional derivatives

$$
\begin{aligned}
\sigma\left(\boldsymbol{r}^{\prime}, \boldsymbol{r}\right) & =-\frac{\delta \rho(\boldsymbol{r})}{\delta u\left(\boldsymbol{r}^{\prime}\right)}=-\frac{\langle\delta \rho \mid \boldsymbol{r}\rangle}{\left\langle\boldsymbol{r}^{\prime} \mid \delta u\right\rangle} \\
& =\left(\frac{\delta \rho(\boldsymbol{r})}{\delta \mu\left(\boldsymbol{r}^{\prime}\right)}\right)_{v}=\left(\frac{\langle\delta \rho \mid \boldsymbol{r}\rangle}{\left\langle\boldsymbol{r}^{\prime} \mid \delta \mu\right\rangle}\right)_{v} \\
& =-\left(\frac{\delta \rho(\boldsymbol{r})}{\delta v\left(\boldsymbol{r}^{\prime}\right)}\right)_{\mu}=-\left(\frac{\langle\delta \rho \mid \boldsymbol{r}\rangle}{\left\langle\boldsymbol{r}^{\prime} \mid \delta v\right\rangle}\right)_{\mu} .
\end{aligned}
$$

In the next section we shall use the $(P, \mathrm{CT})$-projections to extract from the hardness and softness 
operators/kernels the specific components reflecting the pure $P$ or CT effects, and those reflecting the coupling between these two degrees-of-freedom of electronic densities in molecular systems.

\section{Geometric decomposition of the Fukui function}

Equation (47) can be also interpreted as the position representation of the underlying operator equation in the molecular Hilbert space for density displacements:

$$
\hat{\sigma}=-\hat{\beta}+|f\rangle S\langle f| \equiv-\hat{\beta}+S \hat{\mathrm{P}}_{f}
$$

where $|f\rangle$ represents the electronic $\mathrm{FF}$, (46), in the density-displacement vector space: $f(\boldsymbol{r})=\langle\boldsymbol{r} \mid f\rangle$, which defines the associated FF projector $\hat{\mathrm{P}}_{f}=|f\rangle$ $\langle f|$. The complementary $(P, \mathrm{CT})$-projections of the density displacements in the open molecular systems then give rise to the corresponding geometric interpretation of the FF vector:

$$
\begin{aligned}
|f\rangle & =\left(\frac{|\partial \rho\rangle}{\partial N}\right)_{V}=N\left(\frac{|\partial p\rangle}{\partial N}\right)_{V}+|p\rangle \\
& \equiv|f\rangle_{N}+|f\rangle_{C T}=|f\rangle_{N}+\left(\left|p_{C T}\right\rangle+\left|p_{P}\right\rangle\right) \\
& \equiv|f\rangle_{N}+\left[\left|f_{C T}\right\rangle_{\text {hom }}+\left|f_{C T}\right\rangle_{\text {inhom }}\right] \\
& =\left(|f\rangle_{N}+\left|p_{P}\right\rangle\right)+\left|p_{C T}\right\rangle \\
& =\left(|f\rangle_{N}+\left|f_{C T}\right\rangle_{\text {inhom }}\right)+\left|f_{C T}\right\rangle_{\text {hom }} \\
& \equiv|f\rangle_{P}+\left|f_{C T}\right\rangle_{\text {hom }}
\end{aligned}
$$

where the internal, closed system component,

$$
|f\rangle_{N}=\left(\frac{\partial|\rho\rangle_{N}}{\partial N}\right)_{v}=\left(\frac{N \partial|p\rangle}{\partial N}\right)_{v}
$$

is due to a change in the shape factor of the electron distribution, and the external CT-component,

$$
|f\rangle_{C T}=\left(\frac{\partial|\rho\rangle_{C T}}{\partial N}\right)_{v}=|p\rangle
$$

contains both the inhomogeneous (polarizational) component,

$$
\left|f_{C T}\right\rangle_{\text {inhom }}=\left(\frac{\partial\left(\hat{\mathrm{P}}_{P}|\rho\rangle_{C T}\right)}{\partial N}\right)_{v}=\left|p_{P}\right\rangle,
$$

and the homogeneous part,

$$
\left|f_{C T}\right\rangle_{\text {hom }}=\left(\frac{\partial\left(\hat{\mathrm{P}}_{C T}|\rho\rangle_{C T}\right)}{\partial N}\right)_{v}=\left|p_{C T}\right\rangle=1 / V
$$

which reflects the shift in the density normalization due to the a unit change in the system overall number of electrons.

Therefore, as in the case of the density displacement, the identity projection of the FF vector,

$$
\begin{aligned}
|f\rangle & =\left(\hat{\mathrm{P}}_{C T}+\hat{\mathrm{P}}_{P}\right)|f\rangle \equiv\left[|f\rangle_{N}+\left|f_{C T}\right\rangle_{\text {inhom }}\right]+\left|f_{C T}\right\rangle_{\text {hom }} \\
& =|f\rangle_{P}+\left|f_{C T}\right\rangle_{\text {hom }},
\end{aligned}
$$

separates the overall intra-system, $P$-component of an open molecule, $|f\rangle_{P}=|f\rangle_{N}+\left|p_{P}\right\rangle$, from the homogeneous external component, $\left|p_{C T}\right\rangle=1 / V$, (15). They give rise to the associated contributions to the FF in the position representation:

$$
\begin{aligned}
f(\boldsymbol{r}) & =\langle\boldsymbol{r} \mid f\rangle=\langle\boldsymbol{r} \mid f\rangle_{P}+\left\langle\boldsymbol{r} \mid f_{C T}\right\rangle_{h o m}=f_{P}(\boldsymbol{r})+1 / V \\
f_{P}(\boldsymbol{r}) & =\langle\boldsymbol{r} \mid f\rangle_{P}=[f(\boldsymbol{r})]_{N}+p_{P}(\boldsymbol{r}) \\
& =N\left(\frac{\partial p(\boldsymbol{r})}{\partial N}\right)_{V}+\int \gamma\left(\boldsymbol{r}, \boldsymbol{r}^{\prime}\right) p\left(\boldsymbol{r}^{\prime}\right) \mathrm{d} \boldsymbol{r}^{\prime}
\end{aligned}
$$

\section{Geometric decomposition of density- potential kernels}

The density partitioning of (8) and the related geometric $(P, \mathrm{CT})$-projections provide additional tools for interpreting energy changes due to shifts in the electron density. Consider, as an illustrative example, the second differential of the universal density functional $F[\rho] \equiv \mathrm{F}[N p]$ equal to that of $E_{v}[\rho]=$ $E[N, v]$ [see (25a), (26) and (42)]:

$$
\begin{aligned}
\Delta^{(2)} E_{v}[\rho] & =\Delta^{(2)} F[\rho] \\
& =\frac{1}{2} \iint \Delta \rho(\boldsymbol{r}) \eta\left(\boldsymbol{r}, \boldsymbol{r}^{\prime}\right) \Delta \rho\left(\boldsymbol{r}^{\prime}\right) \mathrm{d} \boldsymbol{r} \mathrm{d} \boldsymbol{r}^{\prime} \\
& =-\frac{1}{2} \int \Delta u\left(\boldsymbol{r}^{\prime}\right) \Delta \rho\left(\boldsymbol{r}^{\prime}\right) \mathrm{d} \boldsymbol{r}^{\prime} .
\end{aligned}
$$

It should be also emphasized that this energy change also represents the sum of the first two differentials of the grand-potential 


$$
\begin{aligned}
\Omega[u] & =E[N, v]-\left(\frac{\partial E[N, v]}{\partial N}\right)_{v} N=E[N, v]-N \mu \\
& =\int u(\boldsymbol{r}) \rho(\boldsymbol{r}) \mathrm{d} \boldsymbol{r}+F[\rho]=\Omega_{u}[\rho[u]],
\end{aligned}
$$

which provides the "thermodynamic" potential for the open molecular systems in contact with the external electron reservoir (25b). Indeed, by the Euler equation (25a) the first differential of $\Omega_{u}[\rho]$ vanishes at the equilibrium distribution of electrons and hence

$$
\Delta^{(1+2)} \Omega_{u}[\rho]=\Delta^{(2)} \Omega_{u}[\rho]=\Delta^{(2)} F[\rho] .
$$

One should also observe, that the Euler equation (25a) implies the reciprocal functional dependences between the ground-state density and the relative potential: $\rho=\rho[u]$ and $u=u[\rho]$. This further implies that $F[\rho]=F[\rho[u]] \equiv \widetilde{F}[u]$. Using (30) and (66) gives

$$
\Delta^{(2)} \tilde{F}[u]=\frac{1}{2} \iint \Delta u(\boldsymbol{r}) \sigma\left(\boldsymbol{r}, \boldsymbol{r}^{\prime}\right) \Delta u\left(\boldsymbol{r}^{\prime}\right) \mathrm{d} \boldsymbol{r} \mathrm{d} \boldsymbol{r}^{\prime} .
$$

Using (8a) allows one to separate the closed-system and external $C T$-effects in this second-order energy:

$$
\begin{aligned}
\Delta^{(2)} F[\rho]= & \Delta^{(2)} \mathrm{F}[N p] \\
= & \frac{1}{2}\left[\bar{\eta}(\Delta N)^{2}+2 N \Delta N \int \bar{\eta}(\boldsymbol{r}) \Delta p(\boldsymbol{r}) \mathrm{d} \boldsymbol{r}\right. \\
& \left.+N^{2} \iint \Delta p(\boldsymbol{r}) \eta\left(\boldsymbol{r}, \boldsymbol{r}^{\prime}\right) \Delta p\left(\boldsymbol{r}^{\prime}\right) \mathrm{d} \boldsymbol{r} \mathrm{d} \boldsymbol{r}^{\prime}\right],
\end{aligned}
$$

where:

$$
\begin{aligned}
\left(\frac{\partial^{2} \mathrm{~F}[N p]}{\partial N^{2}}\right)_{p}= & \iint\left(\frac{\partial \rho(\boldsymbol{r})}{\partial N}\right)_{p}\left(\frac{\partial^{2} F[\rho]}{\partial \rho(\boldsymbol{r}) \partial \rho\left(\boldsymbol{r}^{\prime}\right)}\right) \\
& \times\left(\frac{\partial \rho\left(\boldsymbol{r}^{\prime}\right)}{\partial N}\right)_{p} \mathrm{~d} \boldsymbol{r} \mathrm{d} \boldsymbol{r}^{\prime} \\
= & \iint p(\boldsymbol{r}) \eta\left(\boldsymbol{r}, \boldsymbol{r}^{\prime}\right) p\left(\boldsymbol{r}^{\prime}\right) \mathrm{d} \boldsymbol{r} \mathrm{d} \boldsymbol{r}^{\prime} \equiv \bar{\eta},
\end{aligned}
$$

$$
\begin{aligned}
-\left(\frac{\partial u(\boldsymbol{r})}{\partial N}\right)_{p} & \equiv \bar{\eta}(\boldsymbol{r})=-\int \frac{\delta u(\boldsymbol{r})}{\delta \rho\left(\boldsymbol{r}^{\prime}\right)}\left(\frac{\partial \rho\left(\boldsymbol{r}^{\prime}\right)}{\partial N}\right)_{p} \mathrm{~d} \boldsymbol{r}^{\prime} \\
& =\int p\left(\boldsymbol{r}^{\prime}\right) \eta\left(\boldsymbol{r}^{\prime}, \boldsymbol{r}\right) \mathrm{d} \boldsymbol{r}^{\prime}
\end{aligned}
$$

This resolution separates the first contribution, due to $\Delta N$ for constant $p$, which involves the average hardness $\bar{\eta}$, from the last term, due to $\Delta p$ for con- stant $N$, and the two coupling terms containing the average local hardness $\bar{\eta}(\boldsymbol{r})$, which is not equalized throughout the space.

Alternatively, the geometric $(P, C T)$-projections can be used to resolve, for interpretative purposes, the second-order energy of (66). The identity projection $\hat{\mathrm{P}}=\hat{\mathrm{P}}_{C T}+\hat{\mathrm{P}}_{P}=1$ placed between the density displacements and the hardness operator of the Hilbert space interpretation of $\Delta^{(2)} E_{v}[\rho]=\Delta^{(2)} F[\rho]$,

$$
\Delta^{(2)} F[\rho]=-\frac{1}{2}\langle\Delta u \mid \Delta \rho\rangle=\frac{1}{2}\langle\Delta \rho|\hat{\mathrm{P}} \hat{\eta} \hat{\mathrm{P}}| \Delta \rho\rangle,
$$

can be attributed to either the density displacements,

$$
\begin{aligned}
\Delta^{(2)} F[\rho] & =\frac{1}{2}(\langle\Delta \rho| \hat{\mathrm{P}}) \hat{\eta}(\hat{\mathrm{P}}|\Delta \rho\rangle) \\
& =-\frac{1}{2}\langle\Delta u|(\hat{\mathrm{P}}|\Delta \rho\rangle)=-\frac{1}{2}(\langle\Delta u| \hat{\mathrm{P}})|\Delta \rho\rangle,
\end{aligned}
$$

or to the hardness operator itself,

$$
\Delta^{(2)} F[\rho]=\frac{1}{2}\langle\Delta \rho|(\hat{\mathrm{P}} \hat{\eta} \hat{\mathrm{P}})| \Delta \rho\rangle .
$$

The former gives rise to the associated partitioning the density displacements, while the latter amounts to dividing the hardness operator into the four matrix elements

$$
\begin{array}{r}
\hat{\eta}=\sum_{X} \sum_{Y} \hat{\mathrm{P}}_{X} \hat{\eta} \hat{\mathrm{P}}_{Y}=\sum_{X} \sum_{Y} \hat{\eta}_{X, Y}, \\
\left(\hat{\mathrm{P}}_{X}, \hat{\mathrm{P}}_{Y}\right) \in\left\{\hat{\mathrm{P}}_{C T}, \hat{\mathrm{P}}_{P}\right\},
\end{array}
$$

and the associated division of the hardness kernel:

$$
\begin{gathered}
\eta\left(\boldsymbol{r}, \boldsymbol{r}^{\prime}\right)=\left\langle\boldsymbol{r}|\hat{\eta}| \boldsymbol{r}^{\prime}\right\rangle \equiv \sum_{X} \sum_{Y}\left\langle\boldsymbol{r}\left|\hat{\eta}_{X, Y}\right| \boldsymbol{r}^{\prime}\right\rangle=\sum_{X} \sum_{Y} \eta_{X, Y}\left(\boldsymbol{r}, \boldsymbol{r}^{\prime}\right) \\
(X, Y) \in\{P, \mathrm{CT}\} .
\end{gathered}
$$

This operator partitioning resolves the differential of (66) into the $(P, \mathrm{CT})$-resolved contributions:

$$
\begin{aligned}
\Delta^{(2)} F[\rho] & =\frac{1}{2} \iint\langle\Delta \rho \mid \boldsymbol{r}\rangle\langle\boldsymbol{r}|(\hat{\mathrm{P}} \hat{\eta} \hat{\mathrm{P}})| \boldsymbol{r}\rangle\left\langle\boldsymbol{r}^{\prime} \mid \Delta \rho\right\rangle \mathrm{d} \boldsymbol{r} \mathrm{d} \boldsymbol{r}^{\prime} \\
& \equiv \frac{1}{2} \sum_{X} \sum_{Y} \iint \Delta \rho(\boldsymbol{r}) \eta_{X, Y}\left(\boldsymbol{r}, \boldsymbol{r}^{\prime}\right) \Delta \rho\left(\boldsymbol{r}^{\prime}\right) \mathrm{d} \boldsymbol{r} \mathrm{d} \boldsymbol{r}^{\prime}
\end{aligned}
$$

A similar projection of the softness operator partitions it into the four matrix components, 


$$
\hat{\sigma}=\sum_{X} \sum_{Y} \hat{\mathrm{P}}_{X} \hat{\sigma} \hat{\mathrm{P}}_{Y}=\sum_{X} \sum_{Y} \hat{\sigma}_{X, Y}
$$

$$
(X, Y) \in\{P, \mathrm{CT}\} .
$$

In the position representation this operator projection gives rise to the corresponding division of the softness kernel:

$$
\begin{aligned}
\sigma\left(\boldsymbol{r}, \boldsymbol{r}^{\prime}\right) & =\left\langle\boldsymbol{r}|\hat{\sigma}| \boldsymbol{r}^{\prime}\right\rangle \equiv \sum_{X} \sum_{Y}\left\langle\boldsymbol{r}\left|\hat{\sigma}_{X, Y}\right| \boldsymbol{r}^{\prime}\right\rangle \\
& =\sum_{X} \sum_{Y} \sigma_{X, Y}\left(\boldsymbol{r}, \boldsymbol{r}^{\prime}\right) .
\end{aligned}
$$

It also provides the associated partitioning of the differential of (69):

$$
\begin{aligned}
\Delta^{2} \tilde{F}[u] & =\frac{1}{2} \iint\langle\Delta u \mid \boldsymbol{r}\rangle\left\langle\boldsymbol{r}|(\hat{\mathrm{P}} \hat{\sigma} \hat{\mathrm{P}})| \boldsymbol{r}^{\prime}\right\rangle\left\langle\boldsymbol{r}^{\prime} \mid \Delta u\right\rangle \mathrm{d} \boldsymbol{r} \mathrm{d} \boldsymbol{r}^{\prime} \\
& \equiv \frac{1}{2} \sum_{X} \sum_{Y} \iint \Delta u(\boldsymbol{r}) \sigma_{X, Y}\left(\boldsymbol{r}, \boldsymbol{r}^{\prime}\right) \Delta u\left(\boldsymbol{r}^{\prime}\right) \mathrm{d} \boldsymbol{r} \mathrm{d} \boldsymbol{r}^{\prime}
\end{aligned}
$$

The $(P, C T)$-resolved kernels

$$
\eta\left(\boldsymbol{r}, \boldsymbol{r}^{\prime}\right) \equiv\left[\begin{array}{ll}
\eta_{C T, C T}\left(\boldsymbol{r}, \boldsymbol{r}^{\prime}\right) & \eta_{C T, P}\left(\boldsymbol{r}, \boldsymbol{r}^{\prime}\right) \\
\eta_{P, C T}\left(\boldsymbol{r}, \boldsymbol{r}^{\prime}\right) & \eta_{P, P}\left(\boldsymbol{r}, \boldsymbol{r}^{\prime}\right)
\end{array}\right]
$$

and

$$
\sigma\left(\boldsymbol{r}, \boldsymbol{r}^{\prime}\right) \equiv\left[\begin{array}{cc}
\sigma_{C T, C T}\left(\boldsymbol{r}, \boldsymbol{r}^{\prime}\right) & \sigma_{C T, P}\left(\boldsymbol{r}, \boldsymbol{r}^{\prime}\right) \\
\sigma_{P, C T}\left(\boldsymbol{r}, \boldsymbol{r}^{\prime}\right) & \sigma_{P, P}\left(\boldsymbol{r}, \boldsymbol{r}^{\prime}\right)
\end{array}\right],
$$

jointly denoted as $\mathbf{x}\left(\boldsymbol{r}, \boldsymbol{r}^{\prime}\right)=\left\{x_{X, Y}\left(\boldsymbol{r}, \boldsymbol{r}^{\prime}\right)\right\}, x=(\eta, \sigma)$, can be directly expressed in terms of the eigenfunctions $\boldsymbol{b}(\boldsymbol{r})$ of (4). For the diagonal $P$-components one finds:

$$
\begin{aligned}
x_{P, P}\left(\boldsymbol{r}, \boldsymbol{r}^{\prime}\right) & \left.=\left\langle\boldsymbol{r}\left|\hat{\mathrm{P}}_{P} \hat{\mathrm{X}} \hat{\mathrm{P}}_{P}\right| \boldsymbol{r}^{\prime}\right\rangle=\sum_{i} \sum_{j}\langle\boldsymbol{r} \mid\rangle\right\rangle\langle i|\hat{\mathrm{x}}| j\rangle\left\langle j \mid \boldsymbol{r}^{\prime}\right\rangle \\
& =\sum_{i} \sum_{j} b_{i}(\boldsymbol{r}) x_{i, j} b_{j}^{*}\left(\boldsymbol{r}^{\prime}\right),
\end{aligned}
$$

where $x_{i, j}=\iint b_{i}^{*}(\boldsymbol{r}) x\left(\boldsymbol{r}, \boldsymbol{r}^{\prime}\right) b_{j}\left(\boldsymbol{r}^{\prime}\right) \mathrm{d} \boldsymbol{r} \mathrm{d} \boldsymbol{r}^{\prime}$. For the two off-diagonal terms one similarly obtains:

$$
\begin{aligned}
x_{C T, P}\left(\boldsymbol{r}, \boldsymbol{r}^{\prime}\right) & =\left\langle\boldsymbol{r}\left|\left(1-\hat{\mathrm{P}}_{P}\right) \hat{\mathrm{X}}_{P}\right| \boldsymbol{r}^{\prime}\right\rangle \\
& =\left\langle\boldsymbol{r}\left|\hat{\mathrm{x}} \hat{\mathrm{P}}_{P}\right| \boldsymbol{r}^{\prime}\right\rangle-x_{P, P}\left(\boldsymbol{r}, \boldsymbol{r}^{\prime}\right)
\end{aligned}
$$$$
=\sum_{j}\langle\boldsymbol{r}|\hat{\mathrm{x}}| j\rangle\left\langle j \mid \boldsymbol{r}^{\prime}\right\rangle-x_{P, P}\left(\boldsymbol{r}, \boldsymbol{r}^{\prime}\right)
$$$$
=\sum_{j} x_{j}(\boldsymbol{r}) b_{j}^{*}\left(\boldsymbol{r}^{\prime}\right)-x_{P, P}\left(\boldsymbol{r}, \boldsymbol{r}^{\prime}\right),
$$

where $x_{j}(\boldsymbol{r})=\int x\left(\boldsymbol{r}, \boldsymbol{r}^{\prime}\right) b_{j}\left(\boldsymbol{r}^{\prime}\right) \mathrm{d} \boldsymbol{r}^{\prime}$, and

$$
\begin{aligned}
x_{P, C T}\left(\boldsymbol{r}, \boldsymbol{r}^{\prime}\right) & =\left\langle\boldsymbol{r}\left|\hat{\mathrm{P}}_{P} \hat{\mathrm{X}}\left(1-\hat{\mathrm{P}}_{P}\right)\right| \boldsymbol{r}^{\prime}\right\rangle \\
& =\left\langle\boldsymbol{r}\left|\hat{\mathrm{P}}_{P} \hat{\mathrm{X}}\right| \boldsymbol{r}^{\prime}\right\rangle-x_{P, P}\left(\boldsymbol{r}, \boldsymbol{r}^{\prime}\right) \\
& =\sum_{i}\langle\boldsymbol{r} \mid i\rangle\left\langle i|\hat{\mathrm{x}}| \boldsymbol{r}^{\prime}\right\rangle-x_{P, P}\left(\boldsymbol{r}, \boldsymbol{r}^{\prime}\right) \\
& =\sum_{i} b_{i}(\boldsymbol{r}) x_{i}^{*}\left(\boldsymbol{r}^{\prime}\right)-x_{P, P}\left(\boldsymbol{r}, \boldsymbol{r}^{\prime}\right),
\end{aligned}
$$

with $x_{i}^{*}\left(\boldsymbol{r}^{\prime}\right)=\int b_{i}^{*}(\boldsymbol{r}) x\left(\boldsymbol{r}, \boldsymbol{r}^{\prime}\right) \mathrm{d} \boldsymbol{r}$. Finally, the diagonal CT-kernel is obtained by subtracting the above three partial kernels from the total kernel:

$$
\begin{aligned}
x_{C T, C T}\left(\boldsymbol{r}, \boldsymbol{r}^{\prime}\right)=x\left(\boldsymbol{r}, \boldsymbol{r}^{\prime}\right)-x_{C T, P}\left(\boldsymbol{r}, \boldsymbol{r}^{\prime}\right) \\
\\
\quad-x_{P, C T}\left(\boldsymbol{r}, \boldsymbol{r}^{\prime}\right)-x_{P, P}\left(\boldsymbol{r}, \boldsymbol{r}^{\prime}\right)
\end{aligned}
$$

This illustrative geometric partitioning of the softness and hardness kernels should facilitate a better assessment of the relative roles played by the chargetransfer and polarization effects in molecular processes, and an evaluation of the strength of their mutual coupling. It also provides a powerful tool for dividing the associated changes in the electronic energy, which is vital for such a diagnosis and ultimately for an understanding of the physical and chemical implications these components have in diverse density rearrangements in molecules.

\section{Conclusion}

We have developed the projection partitioning technique for separating the polarization and charge transfer components of the familiar indices of the reactivity theory within the conceptual DFT approach. This approach uses the projection operators acting in the vector (Hilbert) space spanned by the independent modes of density displacements. It gives rise to the geometric resolution of the overall density reconstructions in chemical processes, which should bring about a better understanding of the relative importance of the $P$ and CT effects in the bondforming-bond-breaking processes in molecules and reactive systems. The illustrative $(P, \mathrm{CT})$-decomposition of the Fukui function as well as the hardness and 
softness kernels has been carried out. A similar division can be carried out for other reactivity criteria. It allows one to view the relations between the electronic perturbations and nuclear responses of the EP perspective in terms of the separate $P$ - and CT-effects, and generates measures of the coupling between these two components.

\section{References}

1. Hohenberg P and Kohn W 1964 Phys. Rev. B136 864; Kohn W and Sham L J 1965 Phys. Rev. A140 1133

2. Par R G and Yang W 1989 Density functional theory of atoms and molecules (New York: Oxford University Press)

3. Nalewajski R F (ed.) 1996 Density functional theory I-IV, Topics in current chemistry (Berlin: SpringerVerlag) vols 180-183
4. Nalewajski R F, Korchowiec J and Michalak A 1996 Topics current chemistry (Berlin: Springer-Verlag) vol 183 , p. 25

5. Nalewajski R F and Korchowiec J 1997 Charge sensitivity approach to electronic structure and chemical reactivity (Singapore: World-Scientific)

6. Nalewajski R F 2003 Adv. Quantum Chem. 43 119; Nalewajski R F 2002 In Reviews of modern quantum chemistry: A celebration of the contributions of Robert G Parr (ed.) K D Sen (Singapore: World Scientific) vol 2, p. 1071

7. Chattaraj P K and Parr R G 1993 Structure and bonding. In Chemical hardness (ed.) K D Sen, vol. 80, p. 11

8. Geerlings P, De Proft F and Langenaeker W 2003 Chem. Rev. 1031793

9. Parr R G and Yang W 1984 J. Am. Chem. Soc. 106 4049

10. Berkowitz M and Parr R G 1998 J. Chem. Phys. 88 2554

11. Nalewajski R F 2002 Chem. Phys. Lett. 353143

12. Nalewajski R F 1988 Z. Naturforsch. $\mathbf{A 4 3} 65$ 